Cite this: Analyst, 2013, 138, 6494

Received 29th April 2013

Accepted 20th August 2013

DOI: 10.1039/c3an00872j

www.rsc.org/analyst

\title{
Optimization of $\mathrm{Fe}_{3} \mathrm{O}_{4} @ \mathrm{Ag}$ nanoshells in magnetic field-enriched surface-enhanced resonance Raman scattering for malaria diagnosis
}

\author{
Clement Yuen and Quan Liu* \\ The great potential of magnetic field enriched surface enhanced resonance Raman spectroscopy (SERRS) \\ for early malaria diagnosis has been demonstrated previously. This technique is able to detect \\ $\beta$-hematin, which is equivalent to a malaria biomarker (hemozoin) in Raman features, at a concentration \\ of $5 \mathrm{nM}$. In this study, we present the optimization of nanoparticles used in the magnetic field enriched \\ SERRS by tuning the core size and shell thickness of nanoparticles with an iron oxide core and a silver \\ shell ( $\mathrm{Fe}_{3} \mathrm{O}_{4} @ \mathrm{Ag}$ ). The discrete dipole approximation (DDA) model was introduced to investigate the \\ localized electromagnetic field distributions and extinction efficiencies of the aggregate of $\mathrm{Fe}_{3} \mathrm{O}_{4} @ \mathrm{Ag}$ \\ and $\beta$-hematin, in correlation with their magnetic field enriched SERRS performance. We find that the \\ optimal core-shell size of $\mathrm{Fe}_{3} \mathrm{O}_{4} @ \mathrm{Ag}$ leading to the effective aggregation of $\mathrm{Fe}_{3} \mathrm{O}_{4} @ \mathrm{Ag}$ and $\beta$-hematin \\ under an external magnetic field with superior extinction efficiencies is the key to realize highly \\ augmented Raman signals in this strategy. Furthermore, it is noted that the optimized result differs \\ from the case without the external magnetic field to that with the external magnetic field. Therefore, \\ this work demonstrates experimentally and theoretically the potential of tuning the core-shell \\ $\mathrm{Fe}_{3} \mathrm{O}_{4} @ \mathrm{Ag}$ for achieving the efficient magnetic field-enriched SERRS detection of $\beta$-hematin for early \\ malaria diagnosis.
}

\section{Introduction}

Hemozoin is a unique biomarker in human malaria disease with isostructural and paramagnetic properties similar to those of the chemically fabricated $\beta$-hematin. ${ }^{1-3}$ The detection of these crystals (hemozoin or $\beta$-hematin) has been demonstrated by Raman spectroscopy and its variations (e.g. resonance Raman, surface enhanced Raman, and tip-enhanced Raman spectroscopy)..$^{4-7}$ These techniques have the advantages of fast data acquisition, minimal need for labor, and minimal requirement of skilled workers, in comparison to the microscopic examination of a blood smear that is the "gold standard" of malaria diagnosis. ${ }^{8}$

Recently, our group has demonstrated magnetic fieldenriched surface-enhanced resonance Raman spectroscopy (SERRS) for the sensitive detection of $\beta$-hematin by using nanoshells with an iron oxide core and a silver shell, i.e. $\mathrm{Fe}_{3} \mathrm{O}_{4}$ @Ag. Our result shows that the detection limit of this technique in terms of the parasitemia level is comparable to that in early malaria infection at the ring stage, which suggests the great potential of this technique for early malaria diagnosis. ${ }^{8}$ The unique feature in this technique is that resonance

Division of Bioengineering, School of Chemical and Biomedical Engineering, College of Engineering, Nanyang Technological University, 70 Nanyang Drive, Singapore 637457. E-mail: quanliu@ntu.edu.sg; Fax: +65 6791 1761; Tel: +65 63168748
Raman, ${ }^{9}$ surface enhanced Raman, ${ }^{6}$ and the magnetic field enrichment ${ }^{\mathbf{1 0}}$ effects are integrated to give further augmentation of the Raman signal, which allows more sensitive $\beta$-hematin detection (at a concentration of $5 \mathrm{nM}$ ) than each of these aforesaid techniques alone. ${ }^{8}$

Although the optimizations of core and shell size-correlated plasmonic properties (e.g. Raman performance, extinction, and electromagnetic field distributions) for similar plasmonic structures have been studied in other experiments without an external magnetic field, ${ }^{\mathbf{1 1}}$ these core and shell-related properties could be modified due to nanoparticle aggregation induced by the external magnetic field. Moreover, $\beta$-hematin crystals, which are the target analyte generating Raman signals for enhancement, possess an elongated shape, a large size and paramagnetic properties compared to those commonly studied molecules such as Rhodamine 6G (R6G). These factors determine that the optimization of nanoshells for the enhancement of $\beta$-hematin Raman signals would be different from other common Raman molecules. In this study, we explore the variation in the core diameter and shell thickness of $\mathrm{Fe}_{3} \mathrm{O}_{4} @ \mathrm{Ag}$ in relation to the magnetic field-enriched SERRS of paramagnetic $\beta$-hematin crystals to maximize Raman signal enhancement.

In this paper, we present the trends in the magnetic fieldenriched SERRS performance of $\beta$-hematin as a function of the core and shell size of $\mathrm{Fe}_{3} \mathrm{O}_{4} @ \mathrm{Ag}$ nanoshells in comparison to 
that without the magnetic field enrichment strategy. The discrete-dipole approximation (DDA) method, ${ }^{12}$ which is flexible in handling irregular geometries, ${ }^{13}$ is exploited to evaluate the performance of $\mathrm{Fe}_{3} \mathrm{O}_{4} @ \mathrm{Ag}$ with different core-shell sizes in the magnetic field-enriched SERRS technique. The aggregation of nanoshells due to the external magnetic field for a range of core and shell sizes during SERRS measurement is also discussed.

\section{Methods and materials}

\section{Fabrication of core-shell $\mathrm{Fe}_{3} \mathrm{O}_{4} @ \mathrm{Ag}$ nanoparticles}

The $\mathrm{Fe}_{3} \mathrm{O}_{4}$ nanoparticles were fabricated by a coprecipitation technique. ${ }^{14} 0.168 \mathrm{M}$ of $\mathrm{FeCl}_{2} \cdot 4 \mathrm{H}_{2} \mathrm{O}$ (Alfa Aesar, USA) and 0.333 $\mathrm{M}$ of $\mathrm{FeCl}_{3} \cdot 6 \mathrm{H}_{2} \mathrm{O}$ (Alfa Aesar, USA) were prepared and dissolved in deionized water, prior to the introduction of $4 \mathrm{M} \mathrm{NaOH}$ (Sigma-Aldrich, USA) drop-wise to the mixture at $60{ }^{\circ} \mathrm{C}$ under vigorous mixing (15k rpm, SilentCrusherM, Heidolph, Germany). To synthesize $\mathrm{Fe}_{3} \mathrm{O}_{4}$ with three different radii, the addition of $\mathrm{NaOH}$ was stopped at $\mathrm{pH}$ values of 11,13 , and 14, respectively, and the mixture was heated subsequently at $70{ }^{\circ} \mathrm{C}$ for another 1 hour and cooled to room temperature under stirring, followed by separation using a magnet and then washing with deionized water. $16.2 \mathrm{mM}$ of the fabricated $\mathrm{Fe}_{3} \mathrm{O}_{4}$ nanoparticles in $20 \mathrm{ml}$ of ethanol was surface grafted with $0.15 \mathrm{~g}$ of polyacrylic acid (Sigma-Aldrich, USA) in $80 \mathrm{ml}$ of ethanol, prior to sonication (Elma E30H, Elma, Switzerland) for $15 \mathrm{~min}$. $\mathrm{Fe}_{3} \mathrm{O}_{4}$ was separated with a magnet, followed by washing with ethanol. About $2.1 \mathrm{mM}$ of $\mathrm{Fe}_{3} \mathrm{O}_{4}$ were re-dispersed in ethanol and deionized water (80.6: 19.4\% v/v) for $\mathrm{Ag}$ shell synthesis.

The Ag shells were coated by using the seed-growth reduction method. ${ }^{8,15}$ The number of $\mathrm{Fe}_{3} \mathrm{O}_{4}$ nanoparticles used, which was estimated by considering the weight and the size of these $\mathrm{Fe}_{3} \mathrm{O}_{4}$ nanoparticles, was kept identical in each of the following sets. For each size of $\mathrm{Fe}_{3} \mathrm{O}_{4}$, we coated the $\mathrm{Ag}$ shell with three different thicknesses by introducing the following mixtures drop-wise to the aforesaid $\mathrm{Fe}_{3} \mathrm{O}_{4}$ suspension in an ultrasonic bath.

Set 1. $\mathrm{AgNO}_{3}$ (2.8 mM, Merck, USA) was added and mixed for $30 \mathrm{~min}$, prior to introduction of hydroxylamine hydrochloride (4.1 mM, MP biomedical, USA), $\mathrm{NaOH}$ (8.1 mM), in Triton X-100 (Bio-Rad laboratories, USA), ethanol, and deionized water $(0.9: 70.8: 28.3 \% \mathrm{v} / \mathrm{v} / \mathrm{v})$;

Set 2 . The chemicals and their concentrations added were the same as the previous set prior to the introduction of $\mathrm{AgNO}_{3}$ (9.7 $\mathrm{mM})$ in Triton X-100, ethanol, and deionized water (2: $65.3: 32.7 \% \mathrm{v} / \mathrm{v} / \mathrm{v})$.

Set 3. The same procedure as stated in set 2, except that $19.4 \mathrm{mM}$ instead of $9.7 \mathrm{mM}$ of $\mathrm{AgNO}_{3}$ was used during the second-time addition of $\mathrm{AgNO}_{3}$.

Set 4. Additional $\mathrm{AgNO}_{3}$ (9.7 mM) was mixed into set 3, prior to the introduction of Triton $\mathrm{X}-100$, ethanol, and deionized water $(2: 65.3: 32.7 \% \mathrm{v} / \mathrm{v} / \mathrm{v})$.

Finally, mixtures in these sets were washed and a magnet was used to separate the $\mathrm{Fe}_{3} \mathrm{O}_{4} @ \mathrm{Ag}$ nanoparticles. The final nanoparticles were obtained by filtering $(0.2 \mu \mathrm{m}$ supor syringe filters, Pall, USA) a suspension of the $\mathrm{Fe}_{3} \mathrm{O}_{4} @ \mathrm{Ag}$ nanoparticles in $15 \mathrm{ml}$ of methanol.

\section{Raman measurements of analytes}

The preparation of analytes [Rhodamine 6G (R6G) and $\beta$-hematin], the setup for magnetic field-enriched SERRS, and Raman instrumentation were the same as reported in the previous publication. ${ }^{8}$ Briefly, for the SERS measurements of R6G (Sigma-Aldrich, USA), aqueous R6G at concentrations ranging from $10^{-6}$ to $10^{-9} \mathrm{M}$ was adsorbed onto $\mathrm{Fe}_{3} \mathrm{O}_{4} @ \mathrm{Ag}$ nanoshells. In the sample preparation for the SERRS evaluation of $\beta$-hematin with and without magnetic field enrichment, $\beta$-hematin crystals were fabricated by using the same acid-catalyzed method as discussed, ${ }^{8}$ at concentrations ranging from $10^{-3}$ to $10^{-7} \mathrm{M}$ were mixed with an equal volume of $\mathrm{Fe}_{3} \mathrm{O}_{4} @ \mathrm{Ag}$ nanoshells suspension of $0.33 \mathrm{ml}$ and the number of nanoshells was about $5 \times 10^{11}$. These analytes were transferred to a small vial made of aluminum foil for SERS measurements with the bottom of the vial being exposed to a magnetic field of $0.198 \mathrm{~T}$ and a magnetic field gradient of $26.6 \mathrm{~T} \mathrm{~m}^{-1}$. These samples were excited by a $633 \mathrm{~nm}$ laser (Renishaw, UK) with an excitation power of $0.1 \mathrm{~mW}$ that was focused through a microscope objective $(20 \times, \mathrm{NA}=0.4$, Leica) to a spot about $3 \mu \mathrm{m}$ in diameter. The emitted Raman signals were fed into a Czerny-Turner type spectrograph $(f=250 \mathrm{~mm})$ and a holographic grating (1800 $\mathrm{g} \mathrm{mm}^{-1}$ ) dispersed the incoming signal into a RemCam CCD detector (inVia, Renishaw, UK) with a spectral resolution of $2 \mathrm{~cm}^{-1}$. All spectra were acquired with an integration time of $15 \mathrm{~s}$ and averaged from more than five different samples, in which the typical standard deviation of the signal intensity is smaller than $5 \%$ for R6G and smaller than $10 \%$ for $\beta$-hematin. These raw data were corrected for the baseline level, then smoothed by averaging over a window of five points, and processed for removing the fluorescence background to yield the final spectra.

\section{Discrete-dipole approximation (DDA) model}

The discrete-dipole approximation (DDA) $\operatorname{model}^{12,16}$ was used to calculate the extinction efficiency $\left(Q_{\text {ext }}\right)$ of $\mathrm{Fe}_{3} \mathrm{O}_{4} @ \mathrm{Ag}$ nanoparticles with different core and shell dimensions under the excitation wavelength of $633 \mathrm{~nm}$. $Q_{\text {ext }}$ was calculated for comparison with the experimental SERS performance of these nanoparticles, since $Q_{\text {ext }}$ and SERS signals are closely related to the induced electromagnetic field $\left(E_{\text {ind }}\right) \cdot{ }^{12,13} Q_{\text {ext }}$ is related to the induced electromagnetic field by the imaginary (Im) part in the equation, ${ }^{12}$

$$
Q_{\text {ext }}=\frac{4 \pi k}{\left|E_{\mathrm{O}}{ }^{2}\right|} \sum_{j=1}^{N} \operatorname{Im}\left(E_{\text {ind }, j}^{*} P_{j}\right) / a_{\mathrm{eff}},
$$

where $k$ is the wavevector, $E_{\mathrm{O}}$ is the amplitude of the incident electromagnetic field, $E_{\mathrm{inc}, j}$ is the induced electromagnetic field incident on the $j$-th dipole, with a polarization of $P_{j}(j=1,2, \ldots$, $N$ ) for $N$ discretized cube-shaped dipoles with sides of length $5 \mathrm{~nm}$ and $a_{\text {eff }}$ is the effective area. For SERS signals, the intensities can be approximated to be proportional to $\left|E_{\text {ind }}\right|^{4} .^{17}$ Moreover, the electromagnetic field distributions of these $\mathrm{Fe}_{3} \mathrm{O}_{4} @ \mathrm{Ag}$ nanoparticles in close contact with the irregularshaped $\beta$-hematin crystal were investigated. The ADDA code ${ }^{\mathbf{1 6}}$ was used in the DDA calculations with the assumption that 
these nanoparticles were comprised of $\mathrm{Fe}_{3} \mathrm{O}_{4}, \mathrm{Ag}$, and $\beta$ hematin only, using electric constants ${ }^{18-20}$ reported in the literature.

\section{Results}

Fig. 1 shows the field emission scanning electronic microscopy (FESEM, JEOL JSM-6700F, JEOL, Japan) images of the fabricated $\mathrm{Fe}_{3} \mathrm{O}_{4} @ \mathrm{Ag}$ nanoparticles. These $\mathrm{Fe}_{3} \mathrm{O}_{4}$ nanoparticles show radii of (a) 10, (b) 25, and (c) $40 \mathrm{~nm}$ serving as the cores of nanoshells. The cores with a radius of $10 \mathrm{~nm}$ were coated with $10,30,40$, and $50 \mathrm{~nm}$ thick Ag [Fig. 1(a)]. The cores with a radius of $25 \mathrm{~nm}$ were coated with 10, 25, 45 and $60 \mathrm{~nm}$ thick Ag [Fig. 1(b)]. The cores with a radius of $40 \mathrm{~nm}$ were coated with $10,40,50$ and 60 $\mathrm{nm}$ thick Ag [Fig. 1(c)]. The total size typically had a range of less than $\pm 30 \mathrm{~nm}$ according to zetasizer measurements. The compositions of nanoshells were confirmed by taking energydispersive $\mathrm{X}$-ray graphs, which show $\mathrm{Fe}, \mathrm{O}$, and $\mathrm{Ag}$ peaks originated from the $\mathrm{Fe}_{3} \mathrm{O}_{4}$ core and $\mathrm{Ag}$ shell (result not shown).

Fig. 2(a) shows the SERS spectra of aqueous R6G at a concentration of $1 \mu \mathrm{M}$ adsorbed on these $\mathrm{Fe}_{3} \mathrm{O}_{4} @ \mathrm{Ag}$ nanoparticles whose SEM images are shown in Fig. 1 and Fig. 2(b) plots the corresponding R6G Raman peak intensities at 1508 $\mathrm{cm}^{-1}$ (aromatic C-C stretching). R6G adsorbed on these nanoparticles provides different Raman enhancements [Fig. 2(a)], which are revealed by the Raman intensities of prominent peak locations at about $615 \mathrm{~cm}^{-1}$ (C-C-C ring in-plane bending), 775 $\mathrm{cm}^{-1}$ (CH out-of-plane bending), $1310 \mathrm{~cm}^{-1}$ and $1365 \mathrm{~cm}^{-1}$ (C$\mathrm{C} / \mathrm{C}-\mathrm{N}$ stretching), and $1508 \mathrm{~cm}^{-1}$ (aromatic C-C stretching). ${ }^{8}$ For the shell dimensions C40S60, C40S50, C40S40, C40S10, C25S60, C25S45, C25S25, C25S10, C10S50, C10S40, and C10S30, in which the first number indicates the $\mathrm{Fe}_{3} \mathrm{O}_{4}$ core radius and the second number gives the shell thickness, the signal enhancement is roughly $36.0,26.4,6.2,1.6,23.9,7.8,3.9,1.3$, $4.7,4.3$, and 2.2 times higher than that observed for C10S10.

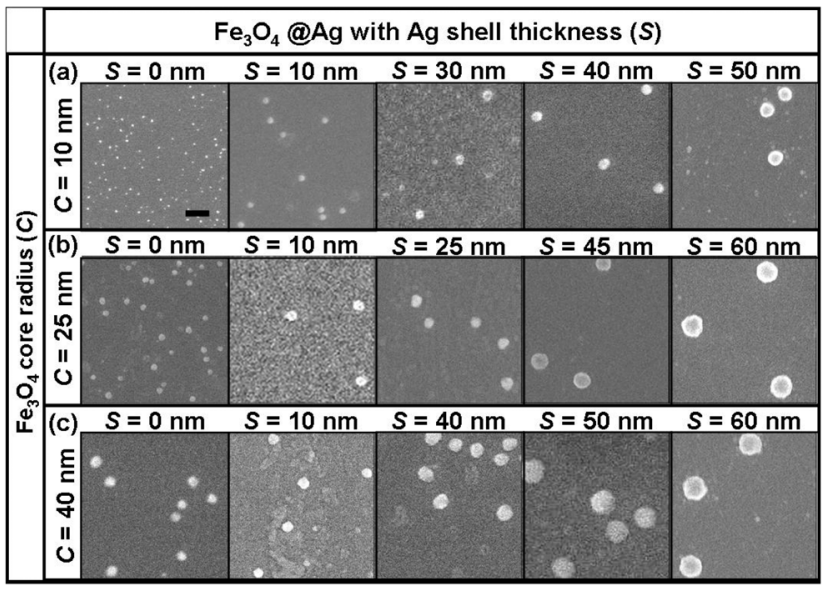

Fig. 1 FESEM images of $\mathrm{Fe}_{3} \mathrm{O}_{4} @ \mathrm{Ag}$ nanoparticles with different $\mathrm{Fe}_{3} \mathrm{O}_{4}$ core radii

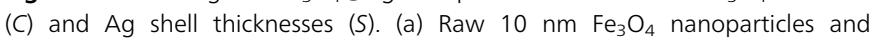
$\mathrm{Fe}_{3} \mathrm{O}_{4} @ \mathrm{Ag}$ with $\mathrm{Ag}$ thicknesses of 10, 30, 40 and 50 nm. (b) Raw 25 nm Fe $\mathrm{O}_{4}$ nanoparticles and $\mathrm{Fe}_{3} \mathrm{O}_{4} @ \mathrm{Ag}$ with $\mathrm{Ag}$ thicknesses of 10, 25, 45 and $60 \mathrm{~nm}$. (c) Raw 40 nm $\mathrm{Fe}_{3} \mathrm{O}_{4}$ nanoparticles and $\mathrm{Fe}_{3} \mathrm{O}_{4} @ \mathrm{Ag}$ with Ag thicknesses of 10, 40, 50 and $60 \mathrm{~nm}$. The scale bar indicates a length of $200 \mathrm{~nm}$ and is shared by all figures.
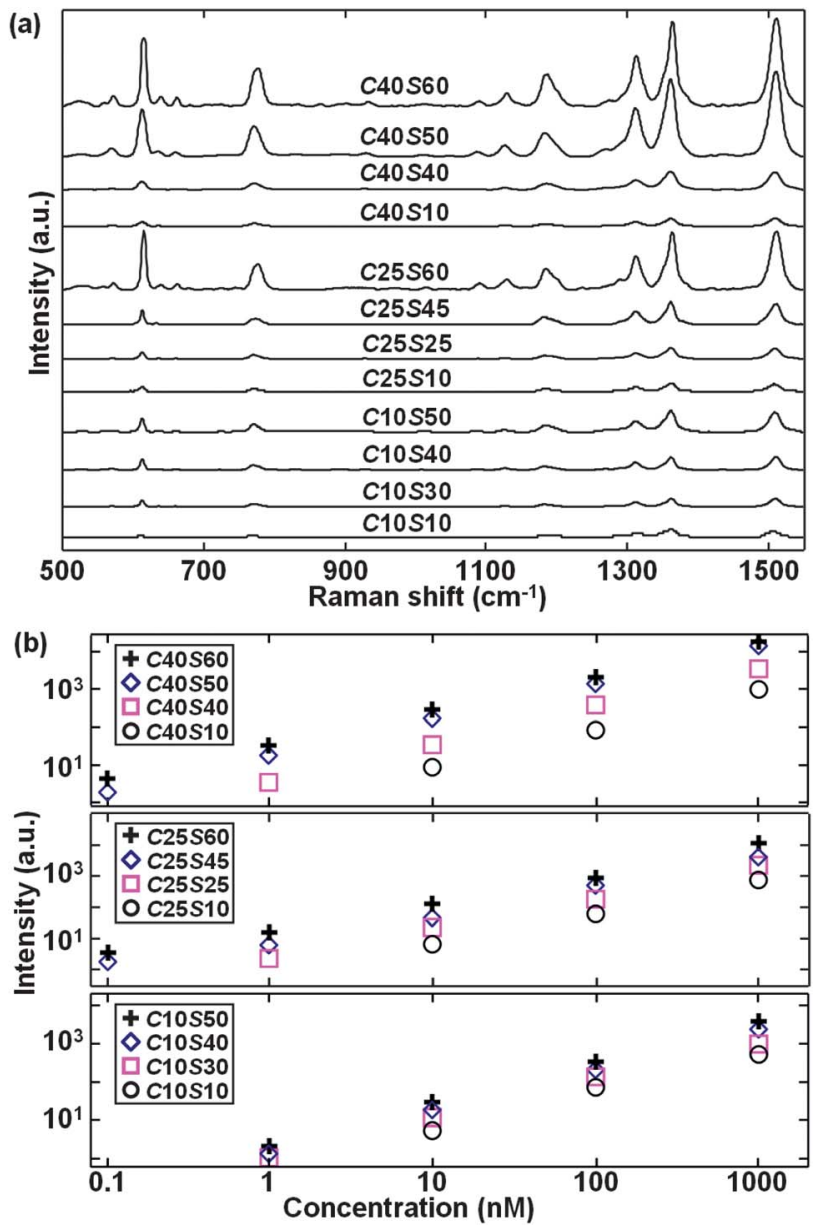

Fig. 2 (a) Experimental SERS spectra of R6G solution at concentrations of $10^{-6}$ $\mathrm{M}$ adsorbed on $\mathrm{Fe}_{3} \mathrm{O}_{4} @ \mathrm{Ag}$ nanoparticles with core-shell parameters of C40S60, C40S50, C40S40, C40S10, C25S60, C25S45, C25S25, C25S10, C10S50, C10S40, C10S30, and C10S10.C10S50 means $\mathrm{Fe}_{3} \mathrm{O}_{4} @ \mathrm{Ag}$ with a core radius of $10 \mathrm{~nm}$ and an outer shell thickness of $50 \mathrm{~nm}$. (b) Raman peak intensities at $1508 \mathrm{~cm}^{-1}$ (aromatic $\mathrm{C}-\mathrm{C}$ stretching) for R6G solution at concentrations of $10^{-6}, 10^{-7}, 10^{-8}$, $10^{-9}$ and $10^{-10} \mathrm{M}$ by using these $\mathrm{Fe}_{3} \mathrm{O}_{4} @ \mathrm{Ag}$ nanoparticles.

These SERS intensities are linearly proportional to the R6G concentrations adsorbed on the $\mathrm{Fe}_{3} \mathrm{O}_{4} @ A g$ with different core and shell sizes [Fig. 2(b)], which are typical ${ }^{13}$ in SERS measurement. The general trend is that a larger $\mathrm{Fe}_{3} \mathrm{O}_{4}$ core and a thicker Ag shell yield higher Raman signal enhancement, with the highest Raman signal enhancement noted for C40S60. The trend in the enhancement factor with the shell and core dimensions of R6G conforms to the typical variation of the core and shell-related SERS performance.

Fig. 3 compares the different enhancement trends in the representative SERRS spectra of $\beta$-hematin (a) without and (b) with the influence of an external magnetic field by using different $\mathrm{Fe}_{3} \mathrm{O}_{4} @ \mathrm{Ag}$ nanoparticles. Interestingly, the trend in magnetic field-enriched SERRS performance is no longer a monotonic function of the core size or shell thickness of $\mathrm{Fe}_{3} \mathrm{O}_{4} @ \mathrm{Ag}$ nanoparticles, based on intensities corresponding to the most prominent vibrational features of $\beta$-hematin, such as $\nu_{15}\left(754 \mathrm{~cm}^{-1}, D_{4 \mathrm{~h}}\right.$ notation system for resonance Raman peak studies on myoglobin $),{ }^{21} \nu_{22}\left(1120 \mathrm{~cm}^{-1}\right), \nu_{2}\left(1570 \mathrm{~cm}^{-1}\right)$, or $\nu_{10}$ 
(a)

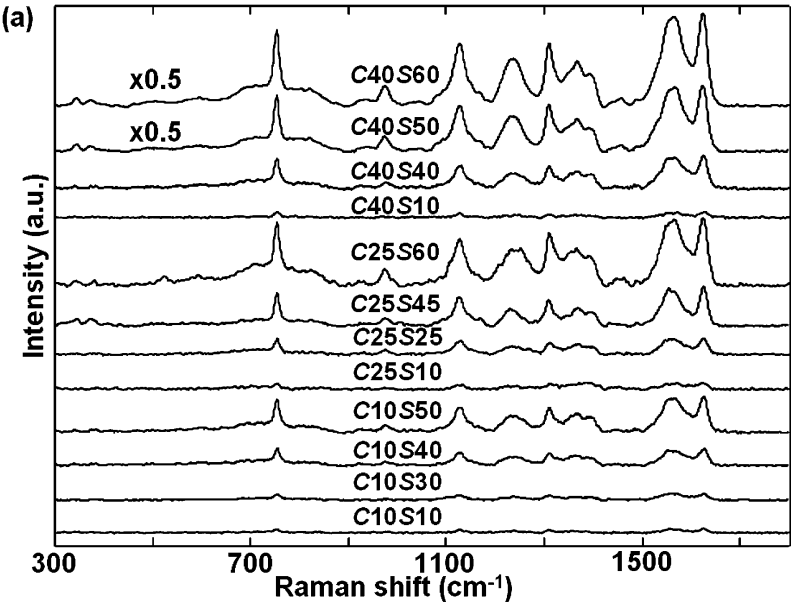

(b)

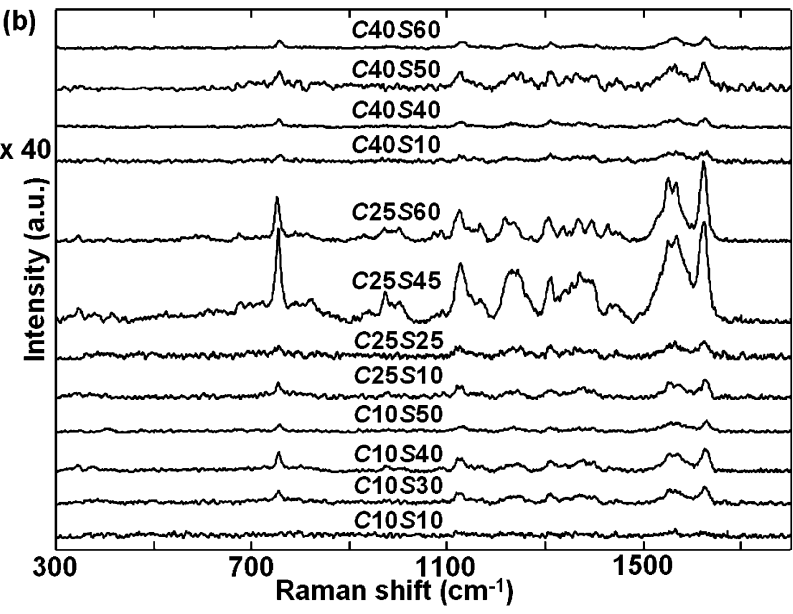

Fig. 3 Experimental SERRS spectra of $\beta$-hematin at (a) concentration of $0.5 \mathrm{mM}$ without magnetic-field enrichment and (b) concentration of $0.5 \mu \mathrm{M}$ with magnetic-field enrichment by using $\mathrm{Fe}_{3} \mathrm{O}_{4} @ \mathrm{Ag}$ nanoparticles of different coreshell parameters. Some spectra are scaled by the specified magnifying factors to facilitate comparison.

$\left(1628 \mathrm{~cm}^{-1}\right)$. Note that these SERS spectra of $\beta$-hematin are similar to RRS spectra reported ${ }^{5}$ and the SERS spectra in tipenhanced Raman measurements of hemozin ${ }^{7}$ without any influence of the magnetic field. Such a similarity can originate from the fact that the free-carboxylate $\mathrm{COOH}$ group in $\beta$-hematin, which is supposed to be responsible for the formation of hydrogen bonds in a physisorption process, ${ }^{22}$ is difficult to attach to the $\mathrm{OH}$-terminated group of Triton $\mathrm{X}-100$ capped $\mathrm{Ag}$ surfaces because the hydrogen bond formed between the $\mathrm{OH}$ group of Triton X-molecules and water molecules is much stronger $^{23}$ than that established with a $\mathrm{COOH}$ group. In contrast, R6G molecules are allowed to chemisorb with the Triton X-100 molecules ${ }^{24}$ in close proximity to the Ag shell for giving rise to stronger adsorption. Thus, attachment of $\mathrm{Fe}_{3} \mathrm{O}_{4} @ \mathrm{Ag}$ to $\beta$-hematin can be enabled by magnetic forces under the influence of a magnetic field instead of weak adsorption. A similar observation, i.e. the weak adsorption of molecules on Ag yields SERRS spectra similar to ordinary Raman spectra, has been reported previously. ${ }^{25}$ The shell dimension of C25S45 yields the largest SERRS signal among
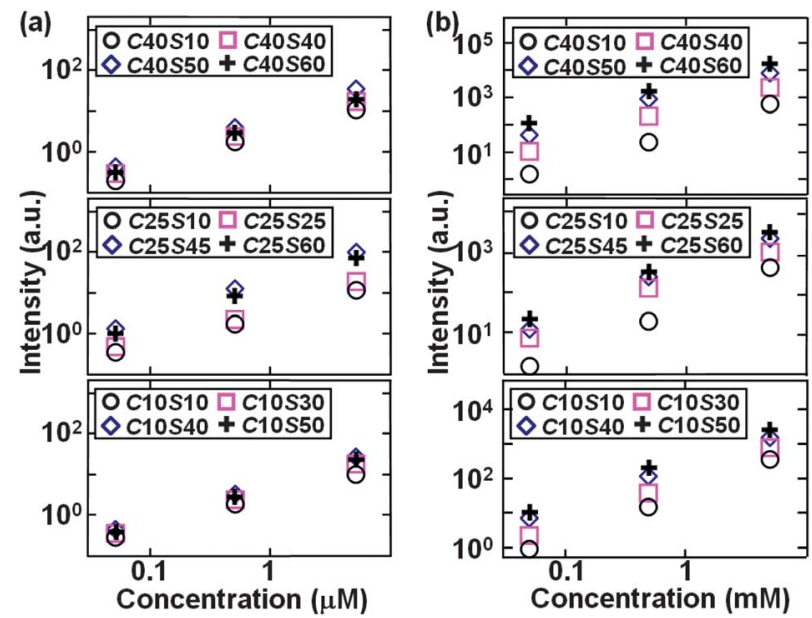

Fig. 4 SERRS intensities at Raman peak $\nu_{10}\left(1628 \mathrm{~cm}^{-1}\right)$ for $\beta$-hematin at $5 \times$ $10^{-3}, 5 \times 10^{-4}, 5 \times 10^{-5}, 5 \times 10^{-6}, 5 \times 10^{-7}$, and $5 \times 10^{-8} \mathrm{M}$ by using $\mathrm{Fe}_{3} \mathrm{O}_{4} @ \mathrm{Ag}$ nanoparticles of different core-shell parameters (a) with and (b) without magnetic enrichment.

these nanoparticles, which is about 10.3 times higher in Raman intensity than that for $C 10 S 10$. Therefore, the core and shell size-correlated enhancement trends of $\mathrm{Fe}_{3} \mathrm{O}_{4} @ \mathrm{Ag}$ are modified in the magnetic field-enriched SERRS measurement of $\beta$-hematin.

Fig. 4 shows the corresponding $\beta$-hematin SERRS intensities at Raman peak $\nu_{10}\left(1628 \mathrm{~cm}^{-1}\right)$ in the case with magnetic-field enrichment in comparison to those without magnetic-field enrichment. The magnetic-field enriched $\beta$-hematin SERRS performance is more sensitive and displays the highest SERRS intensities for C25S45 among these nanoparticles [Fig. 4(a)], which is different from the trends observed without magnetic enrichment [Fig. 4(b) and 2] that show a higher SERRS signal for a larger core. Thus, selecting the optimal core-shell dimensions of $\mathrm{Fe}_{3} \mathrm{O}_{4} @ \mathrm{Ag}$ can improve the magnetic field-enriched SERRS detection of $\beta$-hematin.

\section{Discussion}

The trend in Raman enhancement with different core-shell dimensions shown above can be explained in terms of the extinction efficiencies and magnetic enrichment effectiveness contributed by these $\mathrm{Fe}_{3} \mathrm{O}_{4} @ \mathrm{Ag}$ nanoparticles with different core diameters and shell thicknesses.

Higher SERS enhancement resulted in (1) a thicker Ag shell with the same $\mathrm{Fe}_{3} \mathrm{O}_{4}$ core size (e.g. C40S10, C40S40, C40S60, and $C 40 S 60)$ or (2) a larger core size with the same shell thickness (e.g. C10S10, C25S10, and C40S10) for the R6G SERS (Fig. 2) and $\beta$-hematin SERRS in the case without magnetic enrichment [Fig. 4(b)]. This observation is consistent with the enhancement trends derived from the extinction efficiencies in other types of core-shell nanoparticles reported in the literature. ${ }^{13,26}$ In contrast, $\beta$-hematin SERRS in the case of magnetic-field enrichment [Fig. 3(b) and 4(a)] gives a different trend and an improved sensitivity, in comparison to that without the magnetic field. These findings will be explained as follows. 
One factor that may play an important role in the formation of aggregates between $\mathrm{Fe}_{3} \mathrm{O}_{4} @ \mathrm{Ag}$ nanoshells and $\beta$-hematin as well as among nanoshells could be inter-particle forces. Among these forces, the magnetic dipole-dipole attractive and repulsive force is likely most significant in the presence of a magnetic field due to its much longer effective range than other forces such as steric interaction and electrostatic interaction. ${ }^{27}$ The magnetic force can bring two or more nanoparticles or $\beta$-hematin crystals that are originally out of the critical distance for SERS close enough $(<40 \mathrm{~nm})^{8}$ for effective SERS activities. $\mathrm{Fe}_{3} \mathrm{O}_{4} @ \mathrm{Ag}$ nanoshells with a larger $\mathrm{Fe}_{3} \mathrm{O}_{4}$ core have a larger magnetic dipole moment than that of a smaller core since the dipole moment is proportional to the cube of the core diameter. As a result, these nanoparticles will form aggregates easily along the direction parallel to the magnetic field resulting in increased Raman activities. In addition, the magnetic force can be increased with a decreasing Ag shell thickness because a large Ag shell could shield the magnetic force. From the point of view of increasing the formation of aggregates, nanoshells with a large $\mathrm{Fe}_{3} \mathrm{O}_{4}$ core and a thin $\mathrm{Ag}$ shell are desirable, since the magnetic shielding effect should be minimized with a thinner outer Ag shell (similar to other magnetic nanoparticles ${ }^{28}$ reported in the literature). In our experiment, the shielding effect is nominal for the same diameter iron core with a thicker shell (e.g. C40S10, C40S40, and C40S50 in Fig. 3), which can be attributed to the greater weight that allows faster aggregation settlement at the laser focused spot.

We analyze the electromagnetic field $\left(|E|^{2}\right)$ distribution or the extinction efficiency $\left(Q_{\text {ext }}\right)$ enhancement of these nanoparticles without (Fig. 5) and (Fig. 6 and 7) with the

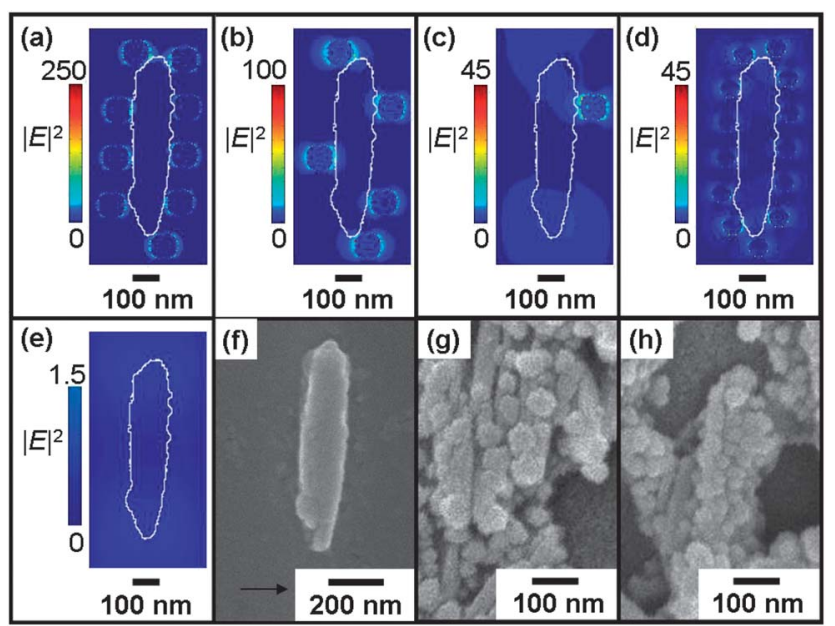

Fig. 5 Calculated electromagnetic $\left(|E|^{2}\right)$ distributions of (a) 9, (b) 5, (c) 1 $\mathrm{Fe}_{3} \mathrm{O}_{4} @ \mathrm{Ag} \mathrm{C} 25 \mathrm{~S} 25$ and (d) $13 \mathrm{Fe}_{3} \mathrm{O}_{4} @ \mathrm{Ag} \mathrm{C} 20 \mathrm{~S} 15$, in contact with the $\beta$-hematin crystal, in comparison with that (e) without $\mathrm{Fe}_{3} \mathrm{O}_{4} @ \mathrm{Ag}$ nanoparticles at $633 \mathrm{~nm}$ wavelength excitation polarized along the horizontal axis. (a) is obtained by introducing four nanoparticles to (b) with the positions of the original five nanoparticles unchanged, and (b) is obtained from (c) likewise. Colors in the color bar have been tuned in (e) to avoid misconception that high $|E|^{2}$ distributions can be achieved without nanoparticles. The white line demarcates the position of a $\beta$ hematin crystal. The topography of the $\beta$-hematin crystal is obtained from (f) the FESEM image. The FESEM image of $\mathrm{Fe}_{3} \mathrm{O}_{4} @ \mathrm{Ag}(\mathrm{g}) \mathrm{C} 25 \mathrm{~S} 25$ and (h) C20S15 with $\beta$ hematin in magnetic field-enrichment.
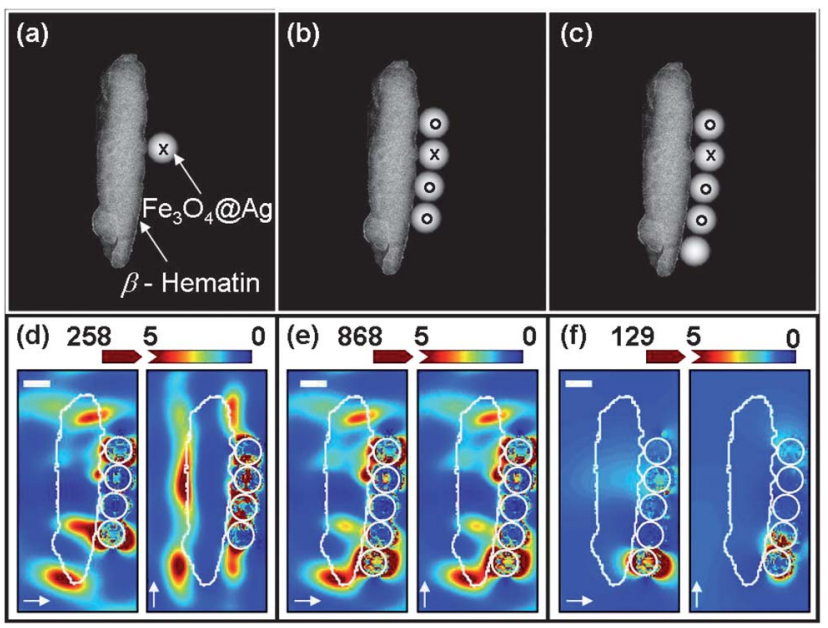

Fig. 6 Schematic diagram used in DDA modeling of $\beta$-hematin and (a) 1, (b) 4, and (c) $5 \mathrm{Fe}_{3} \mathrm{O}_{4} @ \mathrm{Ag}$ nanoparticles (C25S25) at $633 \mathrm{~nm}$ wavelength excitation polarized along the axis as indicated by the arrows. The scale bars represent 100 $\mathrm{nm}$. (d) Ratio of $|E|^{2}$ distributions obtained by a point by point ratio of $|E|^{2}$ distributions between (b) to (a); (e) ratio of $|E|^{2}$ for (c) to (a), and (f) ratio of $|E|^{2}$ for (c) to (b). The intensity in $(d)$ and $(f)$ is rescaled to saturate at higher values for clear illustration of the enhancement at the interface between $\mathrm{Fe}_{3} \mathrm{O}_{4} @ \mathrm{Ag}$ and $\beta$ hematin. The white line outlines the nanoparticles and the $\beta$-hematin.

improvement influenced by neighboring $\mathrm{Fe}_{3} \mathrm{O}_{4} @ \mathrm{Ag}$ based on the DDA method, since Raman intensities are closely related to $|E|^{2}$ and $Q_{\text {ext }}$ as discussed. These coexisting phenomena are illustrated separately for the ease of discussion. For the calculation of $|E|^{2}$ distributions of a $\beta$-hematin crystal in contact with $\mathrm{Fe}_{3} \mathrm{O}_{4} @ \mathrm{Ag}$ (Fig. 5 and 6), the $\beta$-hematin topography is obtained from FESEM [Fig. 5(f)]. A horizontally (Fig. 5 and 6) and vertically (Fig. 6) polarized $633 \mathrm{~nm}$ illumination is excited onto each of the investigated volume of $875 \mathrm{~nm} \times 435 \mathrm{~nm} \times 120$ $\mathrm{nm}$. The $\mathrm{Fe}_{3} \mathrm{O}_{4} @ \mathrm{Ag}$ is modeled as a $\mathrm{Fe}_{3} \mathrm{O}_{4}$ spherical core of diameter $50 \mathrm{~nm}$ with a constant $\mathrm{Ag}$ shell thickness of $25 \mathrm{~nm}$ (unless otherwise stated) to show clearly the dependence of $|E|^{2}$ distribution and $Q_{\text {ext }}$ on the number of $\mathrm{Fe}_{3} \mathrm{O}_{4} @ \mathrm{Ag}$ attached to a $\beta$-hematin.

Fig. 5 shows the $|E|^{2}$ distributions of a $\beta$-hematin in contact with $\mathrm{Fe}_{3} \mathrm{O}_{4} @ \mathrm{Ag}$ spaced at least a radius from each other, to exclude neighboring $\mathrm{Fe}_{3} \mathrm{O}_{4} @ \mathrm{Ag}$ enhancement as shown in our results later. High $|E|^{2}$ intensities are observed at contacts between $\beta$-hematin and $\mathrm{Fe}_{3} \mathrm{O}_{4} @ \mathrm{Ag}$ [Fig. 5(a)-(d)], known as "hot spots", in comparison to the situation without $\mathrm{Fe}_{3} \mathrm{O}_{4} @ \mathrm{Ag}$ nanoparticles [Fig. 5(e)]. The number of hot spots increases with the number of nanoparticles attached to $\beta$-hematin with $Q_{\text {ext }}$ of 4.4, 3.4, and 1.81 for these arrangements as shown in Fig. 5(a)-(c), respectively, which is in agreement with our previous published work $^{8}$ that magnetic enrichment leads to nanoparticle aggregation with better Raman signals. Moreover, we find that smaller $\mathrm{Fe}_{3} \mathrm{O}_{4} @ \mathrm{Ag}\left[\mathrm{C} 20 S 15\right.$ in Fig. 5(d) with $Q_{\text {ext }}$ of 3.4] allows more of the $\mathrm{Fe}_{3} \mathrm{O}_{4} @ \mathrm{Ag}$ to be attached around a $\beta$-hematin crystal [Fig. $5(\mathrm{~g})$ and $(\mathrm{h})]$ to create more hot spots. The comparison between Fig. 5(c) and (d) demonstrates that the increase in the number of hot spots in the arrangement C20S15, as shown in Fig. $5(\mathrm{~d})$, could enlarge $Q_{\text {ext }}$ so that its maximum 


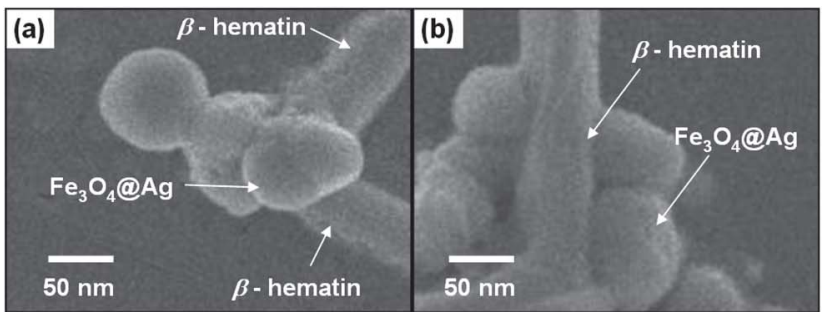

(c)

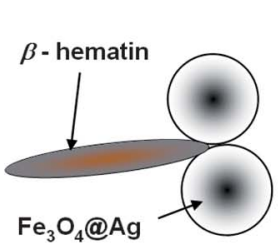

(d)

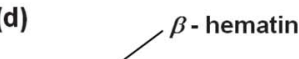

$|E|^{2}$ value is comparable to the arrangement of $C 25 S 25$ in Fig. 5(c), although monomer $C 20 S 15$ shows a lower $Q_{\text {ext }}$ than $C 25 S 25$ theoretically, ${ }^{26}$ due to a smaller core and a thinner shell.

In contrast, Fig. 6 illustrates the ratio of $|E|^{2}$ distributions for different configurations of $\mathrm{Fe}_{3} \mathrm{O}_{4} @ \mathrm{Ag}$ nanoparticles in contact with $\beta$-hematin influenced by the neighboring $\mathrm{Fe}_{3} \mathrm{O}_{4} @ \mathrm{Ag}$ on the original nanoparticle. Real nanoparticle arrangements are simplified to spatial arrangement [e.g. Fig. 6(a)-(c)] to study the enhancement of an electromagnetic field on the original $\mathrm{Fe}_{3} \mathrm{O}_{4} @ \mathrm{Ag}$ (marked with " $\times$ " and "o" at the same position next to the identical $\beta$-hematin) due to additional $\mathrm{Fe}_{3} \mathrm{O}_{4} @ \mathrm{Ag}$ attached to the $\beta$-hematin crystal. We found that additional $\mathrm{Fe}_{3} \mathrm{O}_{4} @ \mathrm{Ag}$ nanoparticles [e.g. Fig. 6(a)-(c)] that are in close contact with each other and attached to a $\beta$-hematin crystal improve the $|E|^{2}$ intensity of the original nanoparticle [Fig. 6(d)(f)]. The $|E|^{2}$ intensity vicinity between the nanoparticle and $\beta$-hematin improves Raman intensity since SERS is approximated to be proportional to $\left|E_{\text {ind }}\right|^{4}$, although reduced $|E|^{2}$ intensity is observed at other locations. These observations imply the same aforesaid point that the magnetic enrichment strategy leads to improved Raman performance for arrangements with more aggregations and smaller $\mathrm{Fe}_{3} \mathrm{O}_{4} @ \mathrm{Ag}$ size.

We also account for the enhancement attributed from other $\mathrm{Fe}_{3} \mathrm{O}_{4} @ \mathrm{Ag}$ located at the ends and opposite sides along the length [Fig. 7(a) and (b)] of the $\beta$-hematin crystal as illustrated in the simplified schematic diagrams in Fig. 7(c) and (d), respectively. Fig. $7(\mathrm{e})$ presents the extinction efficiency $\left(Q_{\text {ext }}\right)$ for different spatial configurations of $\mathrm{Fe}_{3} \mathrm{O}_{4} @ \mathrm{Ag} C 25 S 25$. At our excitation wavelength of $633 \mathrm{~nm}$, a higher $Q_{\text {ext }}$ is observed [Fig. 7(e)] for $\mathrm{Fe}_{3} \mathrm{O}_{4} @ \mathrm{Ag}$ nanoparticle aggregations [Fig. 7(a) and (c)] that form under an external magnetic field in comparison to single-nanoparticle $Q_{\text {ext }}$ which is analogous to dispersed nanoparticles without magnetic enrichment [Fig. 7(e), resonance peak at about $420 \mathrm{~nm}$ typically observed ${ }^{29}$ in individual $\mathrm{Ag}$ nanoparticles]. Moreover, $\mathrm{Fe}_{3} \mathrm{O}_{4} @ \mathrm{Ag}$ positioned on opposite sides of the $\beta$-hematin crystal at about 5 to $40 \mathrm{~nm}$ apart creates high SERS enhancement, which is analogous to other types of SERS nanoparticles reported ${ }^{25}$ in the literature. Thus, further Raman enhancement and dissimilar core and shell size-correlated enhancement trends are noted [Fig. 3(b) and 4(a)] for aggregated nanoparticles.

To briefly summarize our investigation to explain the finding that $C 25 S 45$ yields the largest Raman intensity for $\beta$-hematin SERRS, the following factors would play different roles in the determination of Raman intensity from $\beta$-hematin SERRS measurements.

1. In the absence of a magnetic field, a larger $\mathrm{Fe}_{3} \mathrm{O}_{4}$ core and a larger Ag shell in nanoshells would yield more significant Raman enhancement, which is evident from Fig. 2, 3(a) and $4(\mathrm{~b})$.

2. When an external magnetic field is present, the magnetic attractive force would significantly increase the chance of forming the aggregates of $\beta$-hematin and nanoshells according to the SEM image shown in Fig. 5 and 6 and our previous publication. ${ }^{8}$ This observation could result in two opposing trends.

(a) From the point of view of increasing the formation of aggregates, nanoshells with a large $\mathrm{Fe}_{3} \mathrm{O}_{4}$ core and a thin $\mathrm{Ag}$ shell are desirable according to the discussion earlier about the magnetic force.

(b) However, nanoshells with a smaller size are more likely to create more hot spots around the $\beta$-hematin crystal (Fig. 5) and have much improved extinction (Fig. 6), which would result in higher Raman enhancement.

Thus, the evaluation of the above factors and calculated results (e.g. $|E|^{2}$ distributions and $Q_{\text {ext }}$ ) for the different core and shell dimensions allows tuning of the magnetic field-enriched SERRS, causing C25S45 to give optimal SERRS performance instead of C40S50 in the case of magnetic enrichment. This study provides more insight into SERS performance under an external field for the paramagnetic test molecules and ferromagnetic SERS-active metal nanoshells, which would be otherwise difficult to predict based on the commonly used DDA model alone, since the forecast of the geographical distributions for these molecules and nanoparticles is unknown. The theoretical predictions presented in this work are in agreement with the experimental results (Fig. 3 and 4), which enable the 
nanoshell $C 25 S 45$ to achieve a $\beta$-hematin detection limit of 5 $\mathrm{nM}$ (equivalent to detection of 30 parasites per $\mu \mathrm{l}$ in the earlier malaria stage), as reported in our previous work. ${ }^{8}$

\section{Conclusion}

In conclusion, we report the dependence of magnetic fieldenriched $\beta$-hematin SERRS on the variations of the $\mathrm{Fe}_{3} \mathrm{O}_{4} @ \mathrm{Ag}$ core size and shell thickness. We explain that the magnetic field-enriched SERRS performance of $\beta$-hematin can be further improved by tuning the extinction efficiencies, localized electromagnetic field and magnetic properties of the $\mathrm{Fe}_{3} \mathrm{O}_{4} @ \mathrm{Ag}$ to an optimal core size and shell thickness. Hence, the optimized magnetic field-enriched SERRS performance shows the potential for sensitive $\beta$-hematin detection for early malaria diagnosis. The methodologies developed in this study will be applicable to nanoparticles of other shapes such as nanostars for SERS measurements from $\beta$-hematin, which could yield higher enhancement than nanoshells. This strategy can be also generalized to optimize SERS enhancement for nanocrystals of interest with irregular shapes in other applications.

\section{Acknowledgements}

This research was funded by the Singapore Lee Kuan Yew (LKY) start-up grant. The authors are also grateful to the LKY research fellowship (Clement Yuen) for sponsoring him to carry out his postdoctoral research and the New Investigator Grant (Project no. NMRC/NIG/1044/2011) funded by the National Medical Research Council (NMRC) in Singapore (Quan Liu).

\section{Notes and references}

1 T. J. Egan, J. M. Combrinck, J. Egan, G. R. Hearne, H. M. Marques, S. Ntenteni, B. T. Sewell, P. J. Smith, D. Tayor, D. A. V. Schalkwyk and J. C. Walden, Biochem. J., 2002, 365, 343.

2 D. J. Sullivan, I. Y. Gluzman and D. E. Goldberg, Science, 1996, 271, 219.

3 A. Butykai, A. Orban, V. Kocsis, D. Szaller, S. Bordacs, E. Tatrai-Szekeres, L. F. Kiss, A. Bota, B. G. Vertessy, T. Zelles and I. Kezsmarki, Sci. Rep., 2013, 3, 1431.

4 J. W. Kang, N. Lue, C. R. Kong, I. Barman, N. C. Dingari, S. J. Goldfless, J. C. Niles, R. R. Dasari and M. S. Feld, Biomed. Opt. Express, 2011, 2, 2484.

5 B. R. Wood, A. Hermelink, K. R. Bambery, G. T. Webster, M. A. Khiavi, B. M. Cooke, S. Deed, D. Naumann and D. McNaughton, Analyst, 2009, 134, 1119.
6 S. Cinta-Pinzaru, N. Peica, B. Kustner, S. Schlucker, M. Schmitt, T. Frosch, J. H. Faber, G. Bringmann and J. Popp, J. Raman Spectrosc., 2006, 37, 326.

7 B. R. Wood, E. Bailo, M. A. Khiavi, L. Tilley, S. Deed, T. D. Gaudig, D. McNaughton and V. Deckert, Nano Lett., 2011, 11, 1868.

8 C. Yuen and Q. Liu, J. Biomed. Opt., 2012, 17, 017005.

9 T. Frosch, S. Koncarevic, K. Becker and J. Popp, Analyst, 2009, 134, 1126.

10 D. M. Newman, J. Heptinstall, R. J. Matelon, L. Savage, M. L. Wears, J. Beddow, M. Cox, H. D. Schallig and P. F. Mens, Biophys. J., 2008, 95, 994.

11 S. Lal, S. Link and N. J. Halas, Nat. Photonics, 2007, 1, 641.

12 B. T. Draine and P. J. Flatau, J. Opt. Soc. Am. A, 1994, 11, 1491.

13 C. Yuen, W. Zheng and Z. Huang, J. Raman Spectrosc., 2010, 41, 374.

14 K. T. Wu, P. C. Kuo, Y. D. Yao and E. H. Tsai, IEEE Trans. Magn., 2001, 37, 2651.

15 Y. Zhai, J. Zhai, Y. Wang, S. Guo, W. Ren and S. Dong, J. Phys. Chem. C, 2009, 113, 7009.

16 M. A. Yurkin and A. G. Hoekstra, J. Quant. Spectrosc. Radiat. Transfer, 2011, 112, 2234.

17 C. Yuen, W. Zheng and Z. Huang, J. Innov. Opt. Health Sci., 2008, 1, 267.

18 P. B. Johnson and R. W. Christy, Phys. Rev. B: Solid State, $1972,6,4370$.

19 A. Schegel, S. F. Alvarado and P. Wachter, J. Phys. C: Solid State Phys., 1979, 12, 1157.

20 Y. M. Serebrennikova, J. Patel and L. H. Garcia-Rubio, Appl. Opt., 2010, 49, 180.

21 S. Hu, K. M. Smith and T. G. Spiro, J. Am. Chem. Soc., 1996, 118, 12638.

22 M. T. Shio, F. A. Kassa, M. J. Bellemare and M. Olivier, Microbes Infect., 2010, 12, 889.

23 T. Kar and S. Scheiner, J. Phys. Chem. A, 2004, 108, 9161.

24 D. W. Phillion, D. J. Kuizenga and A. E. Siegman, J. Chem. Phys., 1974, 61, 3828.

25 P. H. B. Aoki, P. Alessio, A. Riul, D. S. Saez and C. J. L. Constantino, Anal. Chem., 2010, 82, 3537.

26 R. Bardhan, N. K. Grady, T. Ali and N. J. Halas, ACS Nano, 2010, 4, 6169.

27 S. Kumar, C. Ravikumar and R. Bandyopadhyaya, Langmuir, 2010, 26, 18320.

28 K. Kim, J. Y. Choi, H. B. Lee and K. S. Shin, ACS Appl. Mater. Interfaces, 2010, 2, 1872.

29 L. Wang, C. Clavero, Z. Huba, K. J. Carroll, E. E. Carpenter, D. Gu and R. A. Lukaszew, Nano Lett., 2011, 11, 1237. 